\section{Influence of the antioxidant content of saliva on dental caries in an at-risk community}

\author{
J. Uberos, ${ }^{1}$ J. A. Alarcón, ${ }^{2}$ M. A. Peñalver, ${ }^{3}$ A. Molina-Carballo, ${ }^{4}$ M. Ruiz, ${ }^{5}$ \\ E. González, ${ }^{6}$ J. Castejon ${ }^{7}$ and A. Muñoz-Hoyos ${ }^{8}$
}

IN BRIEF

- The amount of caries in deciduous teeth is in direct proportion to the observed antioxidant capacity of saliva.

- The total antioxidant capacity of saliva is greater among children that have caries.

- The presencce of caries in deciduous teeth is associated with caries in permanent teeth.

\begin{abstract}
Objective This study aims to evaluate the relationship between the total antioxidant capacity of saliva and the presence of dental caries in deciduous and permanent teeth, in a group of Saharan children. Methods The dental examination was carried out in accordance with the recommendations of the World Health Organization (WHO). The total antioxidant capacity of the saliva was determined by colorimetry. Results The total antioxidant capacity (TAC) of the saliva of patients with caries in deciduous teeth was 2.89 1/IC50 greater than among those without. We observed a statistically significant linear regression between the number of deciduous teeth affected by caries and the total antioxidant capacity of the saliva: $y=0.24+0.53 \times$ TAC saliva $(t=2.93 ; p=0.004)(95 \% \mathrm{Cl}$ of $b: 0.018-0.088)$. Conclusions Our results show that the amount of caries in deciduous teeth is in direct proportion to the observed TAC of saliva, and that the presence of caries in deciduous teeth is associated with caries in permanent teeth.
\end{abstract}

\section{INTRODUCTION}

Dental caries at an early age is still an important problem, with a worldwide incidence ranging from 3-45\%, which in communities that are socioeconomically underprivileged can rise to $70-90 \%{ }^{1}$

Among the factors that have been related to greater cariogenic activity are inadequate dental hygiene and care, the existence of active caries or a family history of caries, a high concentration in the mouth of bacteria with acidophilic activity (Streptococcus mutans or Lactobacillus), reduced salivary flow, a cariogenic diet or inadequate levels of fluoride in drinking water. ${ }^{2}$ Various authors ${ }^{3}$ have shown that fluoride devices release low levels of fluoride for at least two years and have great potential for use in preventing dental caries in high caries risk groups.

\footnotetext{
1*4,8Departamento de Pediatria, Hospital Clínico San Cecilio; 2,3,5,6Facultad de Odontología, Universidad de Granada; ${ }^{7}$ Servicio de Cirugía Infantil, Hospital Virgen de las Nieves, Granada, Spain

*Correspondence to: Dr José Uberos, c/o Málaga, 1. Alfacar 18170 Granada, Spain

Email: joseuberos@telefonica.net
}

\section{Online article number E5}

Refereed Paper - accepted 3 January 2008

DOI: $10.1038 /$ sj.bdj.2008.520

${ }^{\oplus}$ British Dental Journal 2008; 205: E5
Antioxidants constitute an important part of our diet and these, together with intracellular antioxidants and those of the enzymatic systems, can prevent various inflammatory, infectious or tumoural processes. ${ }^{4}$ Of all these mechanisms, particularly important are antioxidant systems of low molecular weight, because these seem to represent the last line of defence against significant cellular lesions. Various authors ${ }^{4,5}$ have examined the relationship between the composition of the saliva and cariogenic activity, the onset and progression of cancer of the tongue or the control of inflammatory processes of the mouth. ${ }^{6}$ A rise in the levels of free radicals has been found to be the common factor in all these processes.

Certain inflammatory processes in the mouth, such as periodontal disease, have been related to an increase in oxidative damage in the mouth and to a decrease in antioxidants. ${ }^{7}$ Moreover, an increase in the antioxidant activity of the saliva has been related to an increase in the suspension of proteins and of cariogenic activity. ${ }^{5}$ However, some authors ${ }^{5}$ found that the differences in total antioxidant capacity (TAC) between caries-active and caries-free subjects were not significant.
Nevertheless, other factors have also been shown to influence its development to a greater or lesser degree. ${ }^{2}$ The real importance of the antioxidant capacity of saliva, as well as the effect of antioxidants in the diet, with respect to the development of caries, remains to be established. ${ }^{4}$ In this sense, it may be of interest to identify the antioxidant state of the saliva and the caries observed in certain ethnic groups with specific, homogeneous patterns of nutrition and hygiene.

We think that the high and continuous levels of antioxidants in saliva contribute to the development of dental caries. The present study aims to evaluate the relationship between the total antioxidant capacity of saliva and the presence of dental caries in deciduous and permanent teeth within a group of Saharan children.

\section{MATERIAL AND METHODS}

\section{Patients}

This study is based on 126 patients from the Western Sahara, resident in a refugee camp at Tindouf (Algeria), of whom $53.8 \%$ were girls (8.5-14.5 years; mean $10.7 \pm 0.19$ [SD]) and $46.2 \%$ boys (4.514-5 years; mean $10.7 \pm 0.28$ [SD]). This 


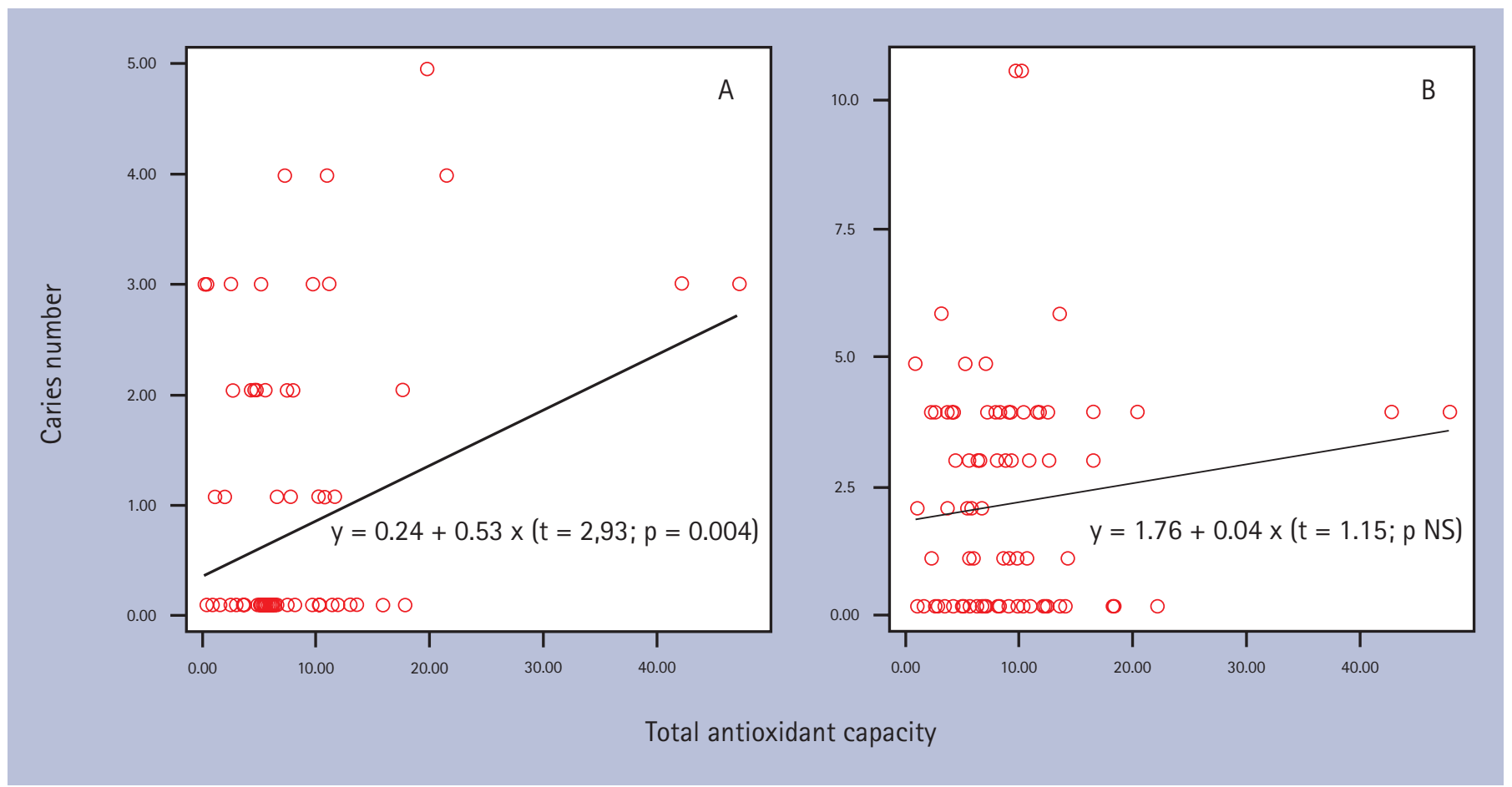

Fig. 1 Regression analysis of the number of caries in primary teeth (A) and in permanent teeth (B) with the total antioxidant capacity of saliva. There is a statistically significant association between the antioxidant capacity of the saliva and the amount of caries in primary teeth

population group provides optimum conditions for the study, given the homogeneity of their social, sanitary, economic and dietary conditions and hygiene customs. In every case, informed consent was requested and obtained from the parent or guardian of the child for their participation in the study. The study was also approved by the local Ethical Committee.

\section{Clinical examination}

This was carried out at the Odontology School of the University of Granada. All the exams were carried out by a specialist doctor in dentistry. The children were examined while staying in Granada on the programme 'vacations in peace' organised by the ONG 'Friends from the Sahara' during the summer of 2005. Clinical exams were carried out, including DMFT (decayed, missing, filled teeth) and the Dean index, which classifies alterations in enamel as very light, light, moderate and severe, in accordance with the classification standards of the World Health Organization. ${ }^{8}$

\section{Evaluation of total antioxidant capacity (TAC)}

Saliva samples were obtained by aspiration during saliva secretion. $2 \mathrm{ml}$ of saliva was obtained and centrifuged at
$4,000 \mathrm{~g}$ for 10 minutes. The supernatant was frozen at $-40^{\circ} \mathrm{C}$ for later use. The TAC of the saliva was determined by colorimetry, in accordance with the technique described by Lussignoli et $a l .{ }^{9}$ The antioxidant power of the sample was characterised as the dose of the substance (or the volume of saliva) which caused a 50\% inhibition of crocin bleaching (IC50). With the test sample we found it useful to express the TAC as the reciprocal of IC50 (TAC $=1 /$ IC50) because in this way the TAC value is directly proportional (and not inversely proportional as in the case when IC50 is used) to the actual antioxidant power of the test sample. All the biological reagents required were supplied by Sigma Chemical Co. (St Louis, MO, USA).

\section{Statistical study}

The results are expressed as the mean \pm standard deviation. We use the value of the median of the variable "caries number' to define the strata of analysis of this variable $(0,1-2,3-4$, and 5 and over caries-active) and the value of the mean + SD. As court point for the TAC of the saliva was considered the value of the mean + SD. The statistical analysis consisted of comparing the means for independent variables, and performing an ANOVA, Pearson correlation analysis and a linear regression study. SPSS 13.0 statistical software was used.

\section{RESULTS}

Thirty-eight percent of the girls had caries in their deciduous teeth, compared with 19\% of the boys. $78.6 \%$ of the children presented with caries in deciduous teeth and $77.8 \%$ in the permanent teeth. The mean (SD) of decayed, missing, filled teeth (DMFT) in deciduous teeth was 0.93 (1.60). The mean (SD) of decayed, missing, filled teeth (DMFT) in permanent teeth was 2.74 (2.83).

The TAC of saliva of the studied group of children was of $8.6 \pm 6.9$ 1/IC50. In children with no caries in their deciduous teeth the TAC of the saliva was 7.8 $\pm 4.01 /$ IC50, while in the caries-active children the TAC was $10.6 \pm 11.11 /$ IC50 $(\mathrm{t}=1.84 ; \mathrm{p}=0.06)$. In children with no caries in their permanent teeth the TAC of the saliva was $7.8 \pm 5.01 /$ IC50 and in the children with caries in the permanent teeth the salivary TAC was 9.0 \pm 8.0 1/IC50. Significant differences do not exist between the groups $(\mathrm{t}=0.82$; $\mathrm{p}=0.41)$. We did not observe statistical differences between the fluorosis levels in the deciduous and permanent teeth and neither did we observe significant association between fluorosis levels and the antioxidants in saliva. 
In children with no caries the TAC of saliva was $7.3 \pm 4.31 /$ IC50, in children with caries activity of $1-2$ the TAC in saliva was $7.2 \pm 4.21 /$ IC50, children with caries activity of 3-4 the TAC was $8.7 \pm$ 4.7 1/IC50 and in children with caries activity of $5+$ the TAC was $11.5 \pm 12.4$ 1/IC50. In patients with levels of TAC in saliva above the mean \pm SD, the risk of presenting with caries in deciduous teeth is OR: 6.25 (95\% CI: 1.47-26.46) and in the permanent teeth is OR: 2.0 (95\% CI: $0.49-8.15)$. Figure 1 shows the regression analysis of salivary TAC and caries.

The salivary TAC of patients with caries in their primary teeth was 2.89 1/IC50 greater than among those without caries. We observed a statistically significant linear regression (Fig. 1) between the number of deciduous teeth with caries and the salivary TAC: $\mathrm{y}=$ $0.24+0.53 \times$ TAC saliva $(\mathrm{t}=2.93 ; \mathrm{p}=$ $0.004)$ (95\% CI of b: 0.018-0.088). In our sample the levels of TAC in saliva did not alter with the age of the children ( $\mathrm{t}=0.83 ; \mathrm{p}=\mathrm{NS})$.

With respect to the permanent teeth, we observed caries in $62 \%$ of the girls and in $58.5 \%$ of the boys. There was no statistical association between caries and the children's sex (OR: 1.16; 95\% CI: 0.50-2.69). We did not observe a significant linear association between the number of permanent teeth affected by caries and the TAC of saliva in these children ( $\mathrm{y}=1.76+0.039 \times$ TAC saliva; $\mathrm{p}=\mathrm{NS})$. Children with caries activity of greater than 5 had a salivary TAC of $11.48 \pm 12.4$ versus corresponding values of $7.79 \pm 4.41$ among the subjects with caries activity of less than $5(\mathrm{t}=$ $2.1 ; p=0.03)$. A statistically significant linear association exists between caries in permanent teeth and the caries in primary teeth $(\mathrm{y}=1.87+0.32 \times$ TAC saliva; $\mathrm{t}=6.89 ; \mathrm{p}<0.001)$.

\section{DISCUSSION}

In previous studies, it has been shown that many bacterial species can modify their adherent activity on epithelial or inert surfaces when the concentration of antioxidants in the medium is altered..$^{10}$ Thus, the development of dental caries will depend on the adherence and growth of cariogenic microorganisms in the dental plaque. Our results show that the amount of caries in deciduous teeth is in direct proportion to the observed TAC of saliva and that the presence of caries in deciduous teeth is associated with caries in permanent teeth.

Saliva is a fluid that contains not just the secretion from salivary glands, but also gingival exudate, epithelial cells, food remains, microorganisms and, sometimes, nasal exudates. ${ }^{11}$ Various authors ${ }^{5}$ have reported higher concentrations of proteins in the saliva of children with greater cariogenic activity; among these children, the concentration of calcium in the saliva is lower than that observed in children with no caries. The concentration of calcium in the saliva increases with age, which would explain the reduction in cariogenic activity in adults, in relation to that observed among children. ${ }^{5}$ These authors do not observe significant statistical differences in the levels of antioxidants in saliva among children with caries and without. We think that these results are influenced by the small number of cases included in each one of the strata of their study $(\mathrm{n}=10)$. In addition, it is not stated whether the children were distributed in each of the strata in a random way. The possibility that a selection bias exists in the sample is therefore high. Although no statistically significant difference was, the tendency of the mean values in that study is toward an increase in antioxidants in the saliva of those subjects with caries.

In our sample fluorosis was not associated with the levels of antioxidants in saliva and fluorosis was similar between deciduous and permanent teeth.

Some authors ${ }^{12}$ have observed an association between prevalence of mutans streptococci in the plaque and caries prevalence. Many dietary factors and some substances of well-known antioxidant effect can influence the adherence of mutans streptococci. ${ }^{13}$

Our findings reveal that the TAC of saliva is greater in patients with caries in deciduous teeth. Taking into account the total caries on deciduous or permanent teeth, we observed that the salivary TAC was significantly higher in patients with caries activity of 5 or more. Due to the age range studied, the exposure time of the teeth to cariogenic agents and to the antioxidant level in saliva is lower for permanent teeth than for primary teeth. We believe this could explain the absence of a statistically significant relationship between the level of antioxidants in saliva and the amount of caries in permanent teeth.

As has been found in previous studies, the adherent activity of some bacteria may be modified by the antioxidant activity of their environment. ${ }^{14,15}$ In this sense, an increase in the TAC of saliva could produce modifications in the adherence of Streptococcus to the dental plaque and lead to greater cariogenic activity. Several authors ${ }^{16}$ have reported greater activity in prooxidant molecules such as nitric oxide (NO) in the saliva of subjects with dental caries. Raised levels of NO in subjects with caries and poor oral hygiene would be a defence mechanism against increased bacterial activity in the mouth. In this respect, higher levels of local antioxidant activity, as has been observed in some of our patients, could counteract the bactericidal effect of the prooxidant molecules present in saliva and on the dental plaque, thus favouring the development of caries. Substances such as polyphenols that are present in various foodstuffs have been shown to have anticariogenic activity, by inhibiting the adherence of Streptococcus mutans to the dental plaque. ${ }^{17}$ This effect seems to depend more upon the inhibitory activity of polyphenols on Streptococcus glucosyltransferase and on the synthesis of glucans than on their antioxidant activity.

From our results, it can be concluded that the TAC of saliva is greater among children that have caries, and that, for the age interval examined and ethnic group in question, the antioxidant capacity of saliva tends to favour the development of dental caries, this effect being more notable on primary teeth.

1. Milnes A R. Description and epidemiology of nursing caries. J Public Health Dent 1996; 56: 38-50.

2. Palmer $\mathrm{C}$. Wolfe $\mathrm{S} \mathrm{H}$. Position of the American Dietetic Association: the impact of fluoride on health. J Am Diet Assoc 2005; 105: 1620-1628.

3. Toumba K J. Slow-release devices for fluoride delivery to high-risk individuals. Caries Res 2001; 35 (Suppl 1): 10-13.

4. Perno Goldie M. Antioxidants in oral health care: making the connection. Int J Dent Hyg 2005; 3: 93-95.

5. Tulunoglu O, Demirtas S, Tulunoglu I. Total antioxidant levels of saliva in children related to 
caries, age, and gender. Int J Paediatr Dent 2006; 16: 186-191.

6. Karincaoglu Y, Batcioglu K, Erdem T, Esrefoglu M Genc M. The levels of plasma and salivary antioxidants in the patient with recurrent aphthous stomatitis. J Oral Pathol Med 2005; 34: 7-12.

7. Brock G R, Butterworth C J, Matthews J B, Chapple I L C. Local and systemic total antioxidant capacity in periodontitis and health. J Clin Periodontol 2004; 31: 515-521.

8. World Health Organization. Oral health surveys - basic methods. 4th ed. Geneva: WHO, 1997.

9. Lussignoli S, Fraccaroli M, Andrioli G, Brocco G Bellavite P. A microplate-based colorimetric assay of the total peroxyl radical trapping capability of human plasma. Anal Biochem 1999; 269: 38-44.

10. Ernster $L$, Forsmark $P$, Nordenbrand $K$. The mode of action of lipid-soluble antioxidants in biological membranes. Relationship between the effects of ubiquinol and vitamin $\mathrm{E}$ as inhibitors of lipid peroxidation in submitochondrial particles. J Nutr SciVitaminol (Tokyo) 1992; Spec No: 548-551.

11. Tenovuo J. Salivary parameters of relevance for assessing caries activity in individuals and populations. Community Dent Oral Epidemiol 1997; 25: 82-86.

12. Seki M, Karakama F, Terajima T et al. Evaluation of mutans streptococci in plaque and saliva: correlation with caries development in preschool children. J Dent 2003; 31: 283-290.

13. Hirasawa M, Takada K, Otake S. Inhibition of acid production in dental plaque bacteria by green tea catechins. Caries Res 2006; 40: 265-270.

14. Uberos J, Augustin C, Liebana J, Molina A, Munoz-
Hoyos A. Comparative study of the influence of melatonin and vitamin $\mathrm{E}$ on the surface characteristics of Escherichia coli. Lett App/ Microbio/ 2001: 32: 303-306.

15. Uberos J, Molina-Carballo A, Galdo-Munoz G, Munoz-Hoyos A. Total antioxidant capacity of plasma in asymptomatic carrier state of Neisseria meningitidis. Epidemiol Infect 2007; 135: 857-860.

16. Bayindir Y Z, Polat M F, Seven N. Nitric oxide concentrations in saliva and dental plaque in relation to caries experience and oral hygiene. Caries Res 2005; 39: 130-133.

17. Yanagida A, Kanda T, Tanabe M, Matsudaira F, Oliveira Cordeiro J G. Inhibitory effects of apple polyphenols and related compounds on cariogenic factors of mutans streptococci. J Agric Food Chem 2000; 48: 5666-5671. 